\title{
The TGFBRI*6A allele is not associated with susceptibility to colorectal cancer in a Spanish population: a case-control study
} Adela Castillejo ${ }^{1}$, Trinidad Mata-Balaguer ${ }^{1}$, Paola Montenegro ${ }^{1}$, Enrique Ochoa ${ }^{3}$, Rafael Lázaro ${ }^{4}$, Ana Martínez-Cantó ${ }^{1}$, MaríaIsabel Castillejo ${ }^{1}$, Carla Guarinos ${ }^{1}$, Víctor-Manuel Barberá1,2, Carmen Guillén-Ponce ${ }^{2}$, Alfredo Carrato ${ }^{1}$ and José-Luís Soto ${ }^{* 1,2}$

Address: ${ }^{1}$ Molecular Oncology Group, Elche University Hospital, Camino Almazara 11, 03203 Elche, Spain, ${ }^{2}$ Genetic Counseling in Cancer Unit, Elche University Hospital, Camino Almazara 11, 03203 Elche, Spain, ${ }^{3}$ Molecular Biopathology Department. Castellon Provincial Hospital. Avenida Doctor Clara 19, 12002 Castellon de La Plana, Spain and ${ }^{4}$ Pathology Department, La Plana Hospital, Partida Carinyena Km 0.5, 12540 Vila-real. Spain

Email: Adela Castillejo - acastillejo@umh.es; Trinidad Mata-Balaguer - trini_mata@hotmail.com; Paola Montenegro - paolacmb@yahoo.es; Enrique Ochoa - enrique.ochoa@hospital2000.net; Rafael Lázaro - lazaro_raf@gva.es; Ana Martínez-Cantó - martinez_anacan@gva.es; MaríaIsabel Castillejo - micastillejo@gmail.com; Carla Guarinos - carlaguarinos@gmail.com; Víctor-Manuel Barberá - barbera_vicjua@gva.es; Carmen Guillén-Ponce - carmenguillenponce@yahoo.es; Alfredo Carrato - acarrato@telefonica.net; José-Luís Soto* - jlsoto@umh.es

* Corresponding author

Published: 18 June 2009

BMC Cancer 2009, 9:193 doi:10.1 186/147|-2407-9-193
Received: 21 January 2009

Accepted: 18 June 2009

This article is available from: http://www.biomedcentral.com//47I-2407/9/193

(c) 2009 Castillejo et al; licensee BioMed Central Ltd.

This is an Open Access article distributed under the terms of the Creative Commons Attribution License (http://creativecommons.org/licenses/by/2.0), which permits unrestricted use, distribution, and reproduction in any medium, provided the original work is properly cited.

\begin{abstract}
Background: TGF- $\beta$ receptor type $I$ is a mediator of growth inhibitory signals. TGFBRI*6A (rs I 1466445) is a common polymorphic variant of the TGF- $\beta$ receptor I gene and has been associated with tumour susceptibility. Nevertheless, the role of this polymorphism as a risk factor for colorectal cancer is controversial. The aim of this study was to assess the association between TGFBRI*6A and colorectal cancer, age, sex, tumour location and tumour stage in a Spanish population.
\end{abstract}

Methods: The case-control study involved 800 Spanish subjects: 400 sporadic colorectal cancer patients and 400 age-, sex-, and ethnic-matched controls. The odds ratio (OR) and $95 \%$ confidence interval $(95 \% \mathrm{Cl})$ for the TGFBRI*6A polymorphism were calculated using unconditional logistic regression adjusted for age and sex. Analysis of somatic mutations at the GCG repeat of TGFBRI exon I and germline allele-specific expression were also conducted to obtain further information on the contribution of the TGFBRI*6A allele to CRC susceptibility.

Results: There was no statistically significant association between the TGFBRI*6A allele and CRC $(p>0.05)$. The OR was I.I47 ( $95 \% \mathrm{Cl}: 0.799-1.647$ ) for carriers of the TGFBRI*6A allele and 0.878 (95\% Cl: $0.306-2.520)$ for homozygous TGFBRI*6A individuals compared with the reference. The frequency of the polymorphism was not affected by age, sex or tumour stage. The TGFBRI*6A allele was more prevalent among colon tumour patients than among rectal tumour patients. Tumour somatic mutations were found in only two of 69 cases $(2.9 \%)$. Both cases involved a GCG deletion that changed genotype $9 A / 9 A$ in normal DNA to genotype $9 A / 8 A$. Interestingly, these two tumours were positive for microsatellite instability, suggesting that these mutations originated because of a deficient DNA mismatch repair system. 


\begin{abstract}
Allele-specific expression of the $9 \mathrm{~A}$ allele was detected in seven of the 14 heterozygous $9 \mathrm{~A} / 6 \mathrm{~A}$ tumour cases. This could have been caused by linkage disequilibrium of the TGFBRI*6A allele with mutations that cause allele-specific expression, as was recently suggested.
\end{abstract}

Conclusion: Our results suggest that the TGFBRI*6A allele does not confer an increased risk of colorectal cancer in the Spanish population.

\section{Background}

Colorectal cancer (CRC) is the main cause of cancerrelated deaths in Spain. Although environmental and genetic factors associated with cancer have been described, current knowledge of the genetic basis of colorectal cancer is limited. About 5\% of colorectal cancer cases are associated with germline mutations of genes such as $A P C$, DNA mismatch repair genes, MYH, SMAD4 and $B M P R 1 A / A L K 3$ [1]. The heritability component of the remaining $95 \%$ of cases is unknown.

Low-penetrance cancer susceptibility alleles may increase the risk of cancer in a manner similar to that of pathogenic germline mutations in hereditary cancer genes. Transforming growth factor beta (TGF- $\beta$ ) is one of the most potent inhibitors of cell growth. TGF- $\beta$ ligands interact with the type II receptor and then bind to the type I receptor, sending a signal to the nucleus through SMAD proteins [2]. Inhibition of TGF function causes unrestricted cell growth because of a lack of growth inhibition. Thus, germline mutations of TGFBR2 and SMAD4 may predispose to the development of hereditary non-polyposis colorectal cancer and juvenile polyposis, respectively [3].

Recently, it has been suggested that a mouse model carrying a germline Tgfbr1 haploinsufficiency has constitutively reduced TGF-beta signalling that significantly enhances the development of colorectal cancer [4].

Polymorphisms of various genes associated with the TGF$\beta$ pathway have been described as cancer risk factors. TGFBR1 ${ }^{*} 6$ A (rs11466445) is a common polymorphic variant of the TGF- $\beta$ receptor I gene. It has been suggested that the TGFBR $1 * 6 A$ allele is a low-penetrance susceptibility factor for colorectal cancer [5-7]. This variant of TGFBR1 is generated by deletion of three GCG triplets that code for three alanine molecules within a nine alanine (*9A) stretch sequence at exon 1 . The TGFBR $1{ }^{*} 6 A$ allele encodes a type I receptor with reduced growth-inhibitory signalling activity [5]. Two meta-analyses, which included 12 case-control studies, have shown that TGFBR $1^{*} 6 \mathrm{~A}$ is a candidate tumour-susceptibility allele, is present in $13.7 \%$ of the general population and increases the risk of cancer by approximately $24 \%[6,7]$. Somatic acquisition of this trinucleotide variant has also been reported in colon cancer, supporting its role as a cancer risk allele [8]. Moreover, it has been proposed that TGFBR ${ }^{*} 6 A$ is responsible for a proportion of patients with hereditary non-polyposis colorectal cancer syndrome [9]. In contrast, based on their case-control study and another meta-analysis [10], Skoglund et al. [10] suggested that this variant is not associated with colorectal cancer. Similarly, others have reported that there is no association between the TGFBR $1{ }^{*} 6$ A allele and prostate [11], lung [12] or bladder [13] cancer. On the other hand, Valle et al. [14] recently suggested that germline allele-specific expression (ASE) of the TGFBR1 gene is dominantly inherited, segregates in families and occurs in sporadic CRC cases. This ASE confers a substantially increased risk of CRC (OR: 8.7; 95\% CI: 2.6-29.1). Moreover, the authors claim that the TGFBR $1{ }^{*} 6$ A allele is probably in linkage disequilibrium with one of the putative mutations that causes ASE, but TGFBR $1{ }^{*} 6$ A per se does not cause ASE. The causative germline changes have not been identified.

Because of this controversy, we conducted a case-control study of the TGFBR $1^{*} 9 A / 6 A$ polymorphism, including mutational and ASE analyses, to clarify the effect of the TGFBR $1 * 6$ A polymorphism on the risk of colorectal cancer in our population.

\section{Methods \\ Subjects}

A total of 400 sporadic CRC cases and 400 controls from the Elche University Hospital and Castellon Provincial Hospital tissue banks were analysed. Written consent to be included in the tissue banks was obtained from each patient. Patients diagnosed with a familial cancer syndrome were excluded.

This was a hospital-based case-control study. Controls with no personal history of cancer, selected with diagnoses considered unrelated to the exposures of interest, were selected from the same hospitals and matched with cases for age, sex and race/ethnicity. The study was approved by the ethical committees of the Elche University Hospital and the Castellon Provincial Hospital.

The median age at diagnosis of CRC was 70 years (range 22-93 years) and that of the controls was 72 years (range 23-98 years). The sex distribution was $41.8 \%$ female $(\mathrm{n}=$ $167)$ and $58.2 \%$ male $(n=233)$ for colorectal cancer patients and $53.2 \%$ female $(n=213)$ and $46.8 \%$ male $(n$ $=187$ ) for the controls. 
Of the CRC cases, 24\% had proximal colon tumours, $33.75 \%$ had distal colon tumours, $9.25 \%$ had sigma-rectum tumours and $25 \%$ had rectal tumours. In $8 \%$ of cases, the location of the tumour was unknown. Cases were classified according to tumour stage as low stage (stages I and II: $78.5 \%$ of cases) or high stage (stages III and IV: $21.5 \%$ of cases) (Table 1).

\section{DNA and RNA samples}

DNA for the case-control study: Control DNA was obtained from peripheral blood samples $(n=400)$. DNA was isolated from the non-tumour colorectal tissue ( $\mathrm{n}=$ 400) of cases after mechanical homogenization (TissueLyser, Qiagen, Valencia, CA). DNA isolation was performed using the EZ1 DNA Tissue kit and the EZ1 BioRobot (Qiagen, Valencia, CA) according to the manufacturers' instructions.

DNA for the TGFBR1 exon 1 tumour mutation: Tumour tissue DNA was isolated after mechanical homogenization of colorectal tumour tissues $(n=69)$ as described previously.

RNA extraction: RNA was isolated from normal colorectal tissue samples from 14 heterozygous 9A/6A CRC patients after mechanical homogenization using the EZ1 RNA Tissue kit and the EZ1 BioRobot (Qiagen, Valencia, CA).

cDNA synthesis: A retrotranscription reaction was performed using Reverse Transcription Reagents, random primers (Applied Biosystems, Foster City, CA) and 200-1000 ng of RNA.

\section{Analysis of the TGFBRI exon I polymorphism, TGFBRI*9A/6A}

Genotyping of the TGFBR1 exon 1 polymorphism was conducted in the case-control study and used for the somatic mutation and ASE analyses. Genotype was assessed using PCR amplification and capillary electrophoresis. The PCR primers were CCACAGGCGGTGGCGGCGGGACCATG (5'-labelled with 6-FAM) for TGFBR1F

Table I: TGFBR I rs I I 466445 genotype frequency according to tumour location and stage (UK: unknown)

\begin{tabular}{cccccc}
\hline Specimens & $\mathbf{n}$ & $\mathbf{9 / 9}$ & $\mathbf{6 / 9}$ & $\mathbf{6 / 6}$ & $\mathbf{P}$ \\
\hline Tumour location & & & & & \\
Proximal colon & 93 & 77 & 15 & $\mathrm{I}$ & \\
Distal colon & 135 & 99 & 34 & 2 & $p=0.109$ \\
Rectum & 100 & 88 & 10 & 2 & $\mathbf{p}=\mathbf{0 . 0 2 4}$ \\
Rectum sigma & 37 & 32 & 4 & $\mathrm{I}$ & \\
UK & 35 & & & & \\
Stage & & & & & \\
I-II (low) & 139 & 108 & 28 & 3 & \\
III-IV (high) & 38 & 31 & 7 & 0 & $p=0.66$ \\
UK & 223 & & & &
\end{tabular}

and CGTCGCCCCCGGGAGCAGCGCCGC for TGFBR1R. PCR amplification was done using the Amplitaq Gold PCR Master Mix $2 \times$ in a total volume of $25 \mu$ l containing $1 \mathrm{M}$ betaine (Sigma Aldrich). The PCR was run for 35 cycles at $94^{\circ} \mathrm{C}$ for $30 \mathrm{~s}$ and $72^{\circ} \mathrm{C}$ for $90 \mathrm{~s}$ followed by a 10 min final extension at $72^{\circ} \mathrm{C}$. PCR products and size standards (Genescan 500 ROX size standard; Applied Biosystems, Foster City, CA) were diluted in HiDi Formamide (Applied Biosystems, Foster City, CA) and then resolved by capillary electrophoresis (ABI 3100 Avant, Applied Biosystems, Foster City, CA) using POP6 as polymer. GeneScan software was used for analysis of PCR fragments (Applied Biosystems, Foster City, CA). Representative cases for each genotype were sequenced directly to confirm allele sizes (data not shown). A PCR product size of 119 bp corresponded to the most common allele, ${ }^{*} 9 A$, whereas a product size of 110 bp corresponded to the ${ }^{*} 6 A$ allele.

\section{Microsatellite instability (MSI) status}

A subset of colorectal tumour DNAs from 120 patients was screened for MSI status using five mononucleotide markers (BAT26, BAT25, NR21, NR24 and NR27) and multiplex PCR as previously described by Buhard et al. [15].

\section{Allele-specific expression of heterozygous *6A $/ 9 A$ individuals}

Fourteen heterozygous 9A/6A CRC cases were included in the ASE analysis. TGFBR1 exon 1 amplicons generated from normal genomic DNA and corresponding normal cDNA were run in parallel. The ASE ratio was calculated by normalizing the ratio between the peak areas of the two alleles in cDNA with the same parameters in genomic DNA. A threshold ratio of a 33\% difference was used to define ASE-positive cases [14].

\section{Statistical analysis}

The Hardy-Weinberg equilibrium was checked among the control and case populations. In the case-control study, we estimated the odds ratio (OR) and 95\% confidence interval $(95 \% \mathrm{CI})$ for the TGFBR1*6A polymorphism using unconditional logistic regression adjusted for age and sex. We analysed for potential effect modification by age using an analysis stratified according to median age at diagnosis ( $\leq 70$ years or $>70$ years). A $\chi^{2}$ test was used to evaluate differences in TGFBR $1^{*} 6 A$ carrier frequencies between the tumour and control groups and to analyse the association between the polymorphism and the clinical and pathological factors. A probability level of $<0.05$ was considered significant.

\section{Results \\ Case-control study}

The genotype distribution in the case and control populations did not deviate significantly from that expected for a 
population in Hardy-Weinberg equilibrium ( $p=0.295$ for cases and $\mathrm{p}=0.033$ for controls). The allele frequencies for the TGFBR $1{ }^{*} 6$ A allele were 0.101 and 0.092 for cases and controls, respectively. Genotype distributions for cases and controls are presented in Table 2. No significant association was observed between the TGFBR ${ }^{*} 6$ A allele and colorectal cancer incidence in our population ( $\mathrm{p}=$ 0.517 ). The crude ORs were 1.147 (95\% CI: 0.799-1.647) for carriers of the TGFBR $1^{*} 6 A$ allele and $0.878(95 \% \mathrm{CI}$ : $0.306-2.520)$ for TGFBR ${ }^{*} 6 A$ homozygous individuals compared with the reference (homozygous TGFBR ${ }^{*} 9 A$ ).

There was no statistically significant association when the OR was adjusted for age and sex. When we analysed the cases according to clinical stage, we detected no significant difference in the TGFBR $1{ }^{*} 6 A$ allele distribution between low and high stages $(\mathrm{p}=0.66)$ (Table 1$)$. On the other hand, TGFBR $1{ }^{*} 6$ A allele carriers were more prevalent among colon (proximal plus distal) cancer patients than rectal cancer patients $(\mathrm{p}=0.024)$ (Table 1$)$.

\section{Frameshift somatic mutation at TGFBRI exon I}

First, we screened colorectal tumour DNA from 120 patients for MSI status. We found evidence of MSI in 14 cases $(14 / 120,11.67 \%)$. Two of 14 MSI tumours (14.29\%) had a deletion of a GCG triplet. All 14 MSI cases were homozygous for the TGFBR ${ }^{*} 9 A$ allele. Both mutated cases were genotyped as heterozygous $9 A / 8 A$. These results were confirmed by direct sequencing (Figure 1 ). To determine whether these somatic mutations are specific for MSI tumours, we analysed tumour DNA from 55 cases that were homozygous for the TGFBR $1^{*} 9 A$ allele and were MSI-negative. None of these 55 cases had a mutation of this GCG repeat ( $\mathrm{p}=0.039$; two-tailed chi square test).

\section{Allele-specific expression in heterozygous $* 6 A / * 9 A$ individuals}

In the genotyping experiments, comparisons between peak areas generated from heterozygous individuals from gDNA and cDNA enabled us to obtain information about the principally expressed allele and the relative level of its expression. Seven of 14 cases had differences greater than $33 \%$ and were considered ASE-positive. Interestingly, all seven cases with ASE over-expressed the TGFBR $1{ }^{*} 9 A$ allele (Figure 2).

\section{Discussion}

There is evidence that common variants of the TGF- $\beta$ pathway alter TGF- $\beta$ signalling and modify cancer risk. The TGFBR1* $6 A$ variant has been associated with breast, ovarian and haematological malignancies [6,9]. Recent studies suggest that the association of the TGFBR $1{ }^{*} 6 A$ allele with colon cancer is either weak (OR: 1.2; 95\% CI: 1.01-1.43) [7] or of borderline significance (OR: 1.13; 95\% CI: 0.98-1.30) [10].

In association studies, contradictory conclusions may arise from population stratification or inappropriate sample size. We determined a priori that our study with 400 sporadic CRC cases and 400 matched controls would enable us to detect an OR of 2.0 for $9 A / 6 A$ heterozygous individuals (assuming a frequency of 0.15 in controls) and an OR of 3.0 for $6 A / 6 A$ homozygous individuals (assuming a frequency of 0.0175 in controls) with $80 \%$ power (twosided test, alpha level $=5 \%$ ).

Our results did not show a statistically significant association ( $\mathrm{p}=0.517$; OR: 1.147 ; 95\% CI: 0.799-1.647), which is in agreement with Skoglund et al. [10]. To our knowledge, the only previous study of this polymorphism in a Spanish population is one that was included in the metaanalysis of Pasche et al. [7]. That case-control study was performed with 298 controls, 237 sporadic CRC cases and 275 sporadic breast cancers. When data for both types of tumour were pooled, a significant increase in cancer risk was associated with TGFBR1 *6A carriers (OR: $1.60 ; 95 \%$ CI: $1.10-2.31)$. The study did not provide information specifically about CRC or about sex matching between the groups. Zhang et al. (2005) suggested that the 12 casecontrol studies of Pasche [7] had marginally significant heterogeneity when pooled and questioned the association between $T G F B R 1^{*} 6 A$ and increased risk of cancer [16]. Similar doubts have been raised in various studies involving different types of tumours [11-13] and in a meta-analysis that included eight case-control studies [10].

Table 2: TGFBR I rs I 4666445 genotype frequency in cases and controls in Spain

\begin{tabular}{|c|c|c|c|c|}
\hline TGFBR I exon I genotype & $\begin{array}{c}\text { Cases } \\
(n=400) \\
\text { number }(\%)\end{array}$ & $\begin{array}{c}\text { Controls } \\
(n=400) \\
\text { number }(\%)\end{array}$ & OR (95\%Cl) & $p$ \\
\hline $9 \mathrm{~A} / 9 \mathrm{~A}$, & $325(81.25)$ & $333(83.25)$ & I (ref.) & \\
\hline $6 \mathrm{~A} / 9 \mathrm{~A}$ & $69(17.25)$ & $60(15)$ & $1.178(0.808-1.718)$ & 0.441 \\
\hline $6 \mathrm{~A} / 6 \mathrm{~A}$ & $6(1.5)$ & $7(1.75)$ & $0.878(0.306-2.520)$ & 1 \\
\hline $6 \mathrm{~A} / *$ & $75(18.75)$ & $67(16.75)$ & 1.147 (0.799-1.647) & 0.517 \\
\hline
\end{tabular}


a)
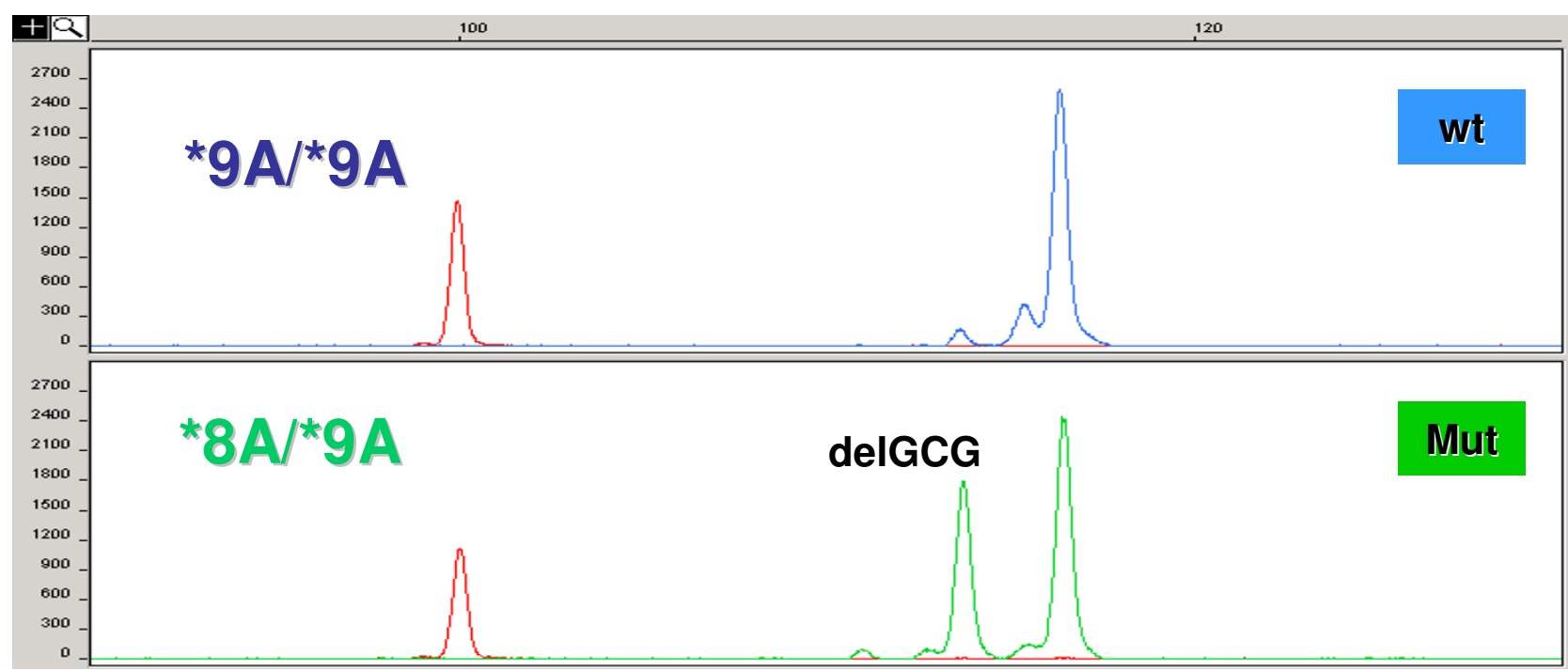

b)

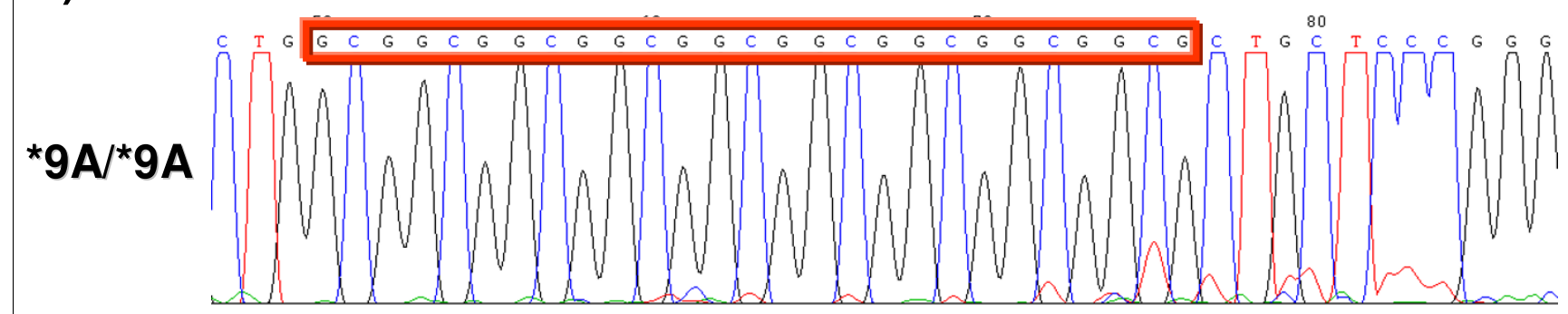

$* 8 A * 9 A$

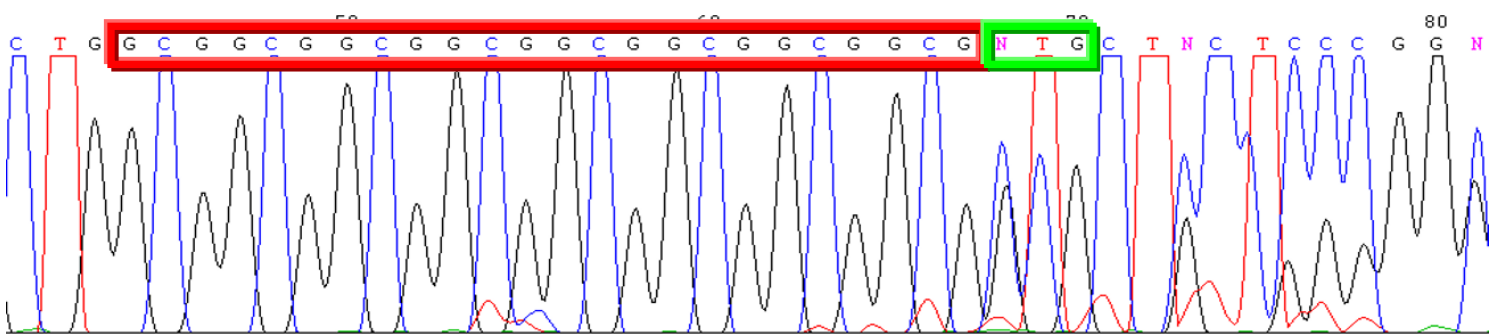

Figure I

Somatic mutation at exon I of the TGFBRI gene in MSI tumours. A GCG deletion of the triplet repeat was found in two independent cases: a) a PCR fragment resolved by capillary electrophoresis, b) DNA sequence from a wild-type case (*9A/ $* 9 A)$ and a mutated case $(* 8 A / * A)$. 


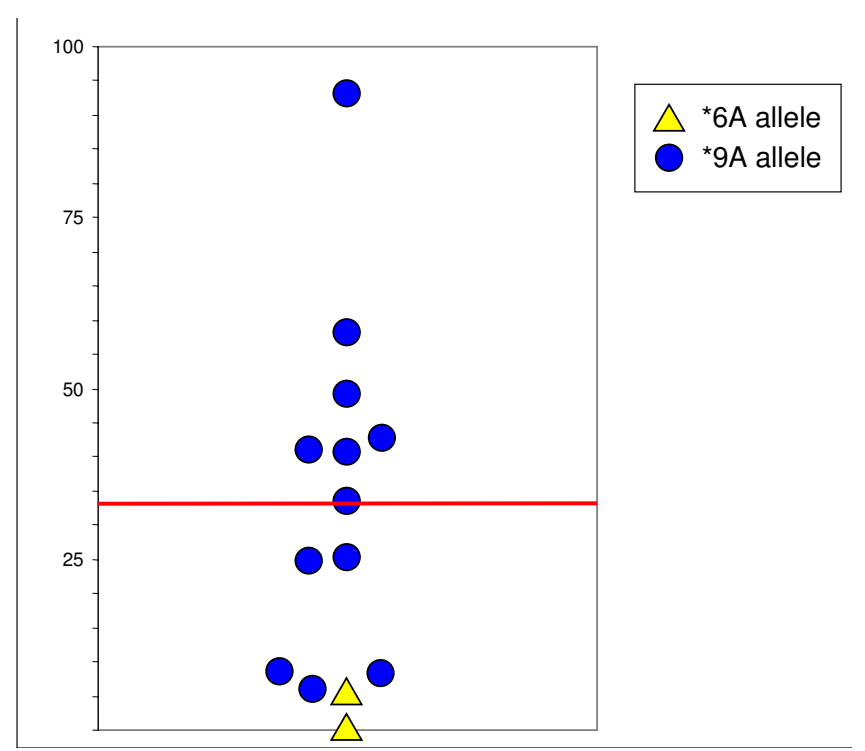

Figure 2

Allele-specific expression of heterozygous $* 6 A / * 9 A$ individuals. The over-expressed allele and the ratio of overexpression are presented. The ASE threshold was defined as $33 \%$.

There was little information on which to base the sample size for our study because information about this polymorphism in our population is limited and contradictory results were reported for another population. The results show that our study was under-powered for detecting weak associations between the TGFBR $1 * 6 \mathrm{~A}$ allele and CRC. The data indicate that a sample size of 10,000 cases and 10,000 controls is necessary to detect an OR of 1.15 with $80 \%$ power using a twosided test with an alpha level of 5\%.

In the present work, age and sex adjustment did have any effect on the risk conferred by the TGFBR $1^{*} 6 A$ allele. Previous case-control studies provide little information in this regard.

Interestingly, there was a significant difference in the frequency of the TGFBR $1{ }^{*} 6 A$ allele between colon and rectal cancer cases. Moreover, when only colon cancer cases where considered, there was a difference of borderline significance for TGFBR $1{ }^{*} 6$ A carriers $(\mathrm{p}=0.071)$ with an OR $=1.47$ (95\% CI: $0.979-2.203)$. No data are available regarding the risk of colon versus rectal cancer for the TGFBR $1{ }^{*} 6$ A allele because data from colorectal and colon cancer studies were pooled in the meta-analyses. Further studies with larger samples are needed to determine if the variant allele is associated specifically with colon cancer but not with rectal cancer. If confirmed, this may help explain the contradictory results in the literature.

Because of the limitation that sample size exerts on association studies, we attempted to extract extra information from our data to elucidate the susceptibility effect of the minor allele of this polymorphism. First, we wanted to know if the GCG repeat is a specific target for frameshift mutations in MSI colorectal tumours, and in the event, if somatic acquisition of TGFBR $1{ }^{*} 6$ A allele occurs in these MSI tumours, similar to metastatic CRC [8]. The underlying hypothesis was that if the minor allele in the germline predisposes to CRC, it might be prevalent in the tumours themselves through somatic acquisition. Our results show that the GCG repeat at exon 1 of the TGFBR1 gene is a specific target for MSI tumours $(\mathrm{p}=0.039)$, but the frequency of mutations is low $(2 / 14,14.29 \%)$ [17]. Nevertheless, there was no somatic acquisition of the TGFBR $1^{*} 6 \mathrm{~A}$ allele. Both mutated cases underwent the deletion of just one GCG triplet. No previous studies have documented the association between this polymorphism and MSI status. Unfortunately, our results did not enable us to clarify the role of the TGFBR $1{ }^{*} 6 A$ allele in CRC risk.

An additional strategy was used to address the role of the minor allele for this polymorphism in CRC. We tested whether gene expression level differed between the alleles of the polymorphism because previously reported data from in vitro models shows that growth inhibitory signalling activity is low for the type I receptor coded by the TGFBR $1{ }^{*} 6 A$ allele [5]. We hypothesized that if predominant expression of the TGFBR ${ }^{*} 6 A$ allele exists in the $6 A /$ $9 A$ heterozygous individual, global functional reduction of the pathway would be evident and would increase the risk for CRC. Surprisingly, the results of the ASE study were opposite to what we had expected. The proportion of ASE positive cases was high (7/14), and there was predominant expression of the TGFBR $1 * 9 A$ allele in all seven ASE positive cases. The correct interpretation of our results became evident after the recent publication of the study of Valle et al. [14]. The authors concluded that ASE of TGFBR1 is a major contributor to genetic predisposition for CRC (OR: 8.7; 95\% CI: 2.6-29.1). They were unable to determine the causative mechanism of the ASE but the haplotype data suggested that ancestral mutations were implicated. One of the putative mutations that causes ASE is probably in linkage disequilibrium with the TGFBR $1{ }^{*} 6$ A allele but is not itself causative of ASE. Our results are in agreement with those of Valle et al. in that about $50 \%$ of $6 \mathrm{~A} / 9 \mathrm{~A}$ heterozygous individuals have ASE and the $6 \mathrm{~A}$ allele is expressed at a low level in all cases. The linkage disequilibrium between ASE and the TGFBR1 ${ }^{*} 6 A$ allele could explain the contradictory results in the literature on the association between this polymorphism and CRC.

\section{Conclusion}

The case-control study did not show a statistical association between the TGFBR $1{ }^{*} 6 A$ allele and CRC, age, sex or tumour stage. The differences in haplotype frequencies between colon and rectal tumour patients should be con- 
firmed by larger series. The somatic mutations that affected the GCG repeat in this study are a consequence of deficient MMR function. The ASE results suggest that there is linkage disequilibrium between the TGFBR ${ }^{*} 6$ A allele and the mutations that cause ASE. Taken together, our results strongly suggest that the TGFBR $1^{*} 6$ A allele does not confer an increased risk of colorectal cancer in the Spanish population.

\section{Competing interests}

The authors declare that they have no competing interests.

\section{Authors' contributions}

$\mathrm{AC}$ participated in the design and coordination of the study, in the molecular genetic studies and helped to draft the manuscript. TM-B, AM-C, M-IC, CG and V-MB participated in the molecular genetic studies and helped to draft the manuscript. PM participated in the design of the study, performed the statistical analysis and helped to draft the manuscript. EO and RL participated in the biobanking of samples and helped to draft the manuscript. CG-P and AC participated in the design of the study and performed the statistical analysis. J-LS conceived the study, participated in its design and coordination and drafted the manuscript. All authors read and approved the final manuscript.

\section{Acknowledgements}

This trial was performed within the cooperative framework established by the Transversal Cancer Action approved by the Council of Ministers on October II, 2007, in accordance with an agreement between the Carlos III Health Institute, which is an autonomous entity currently belonging to the Ministry of Science and Innovation, and the Biomedical Research Foundation of the Hospital of Elche. The research was supported in part by grants from the Generalitat Valenciana in Spain (API06/06) and the Biomedical Research Foundation of the Hospital of Elche (FIBElx-02/2007). T.M-B was a recipient of a fellowship from the Spanish Society of Medical Oncology.

\section{References}

I. Lynch HT, de la Chapelle A: Hereditary colorectal cancer. N Engl J Med 2003, 348:919-932.

2. Derynck R, Akhurst RJ, Balmain A: TGF-beta signaling in tumor suppression and cancer progression. Nat Genet 200I, 29:117-129.

3. Sayed MG, Ahmed AF, Ringold JR, Anderson ME, Bair JL, Mitros FA, Lynch HT, Tinley ST, Petersen GM, Giardiello FM, Vogelstein B, Howe JR: Germline SMAD4 or BMPRIA mutations and phenotype of juvenile polyposis. Ann Surg Oncol 2002, 9:90I-906.

4. Zeng Q, Phukan S, Xu Y, Sadim M, Rosman DS, Pennison M, Liao J, Yang GY, Huang CC, Valle L, Di Cristofano A, de la Chapelle A, Pasche $B$ : TgfbrI haploinsufficiency is a potent modifier of colorectal cancer development. Cancer Res 2009, 69:678-686.

5. Pasche B, Kolachana P, Nafa K, Satagopan J, Chen YG, Lo RS, Brener $D$, Yang D, Kirstein L, Oddoux C, Ostrer H, Vineis P, Varesco L, Jhanwar S, Luzzatto L, Massagué J, Offit K: TbetaR-I(6A) is a candidate tumor susceptibility allele. Cancer Res 1999, 59:5678-5682.

6. Kaklamani VG, Hou N, Bian Y, Reich J, Offit K, Michel LS, Rubinstein WS, Rademaker A, Pasche B: TGFBRI*6A and cancer risk: a meta-analysis of seven case-control studies. J Clin Oncol 2003, 21:3236-3243.

7. Pasche B, Kaklamani V, Hou N, Young T, Rademaker A, Peterlongo P, Ellis N, Offit K, Caldes T, Reiss M, Zheng T: TGFBRI*6A and can- cer: a meta-analysis of 12 case-control studies. J Clin Oncol 2004, 22:756-758.

8. Pasche B, Knobloch TJ, Bian Y, Liu J, Phukan S, Rosman D, Kaklamani V, Baddi L, Siddiqui FS, Frankel W, Prior TW, Schuller DE, Agrawal A, Lang J, Dolan ME, Vokes EE, Lane WS, Huang CC, Caldes T, Di Cristofano A, Hampel H, Nilsson I, von Heijne G, Fodde R, Murty VV, de la Chapelle A, Weghorst CM: Somatic acquisition and signaling of TGFBRI*6A in cancer. JAMA 2005, 294: I634-1646.

9. Bian Y, Caldes T, Wijnen J, Franken P, Vasen H, Kaklamani V, Nafa K, Peterlongo P, Ellis N, Baron JA, Burn J, Moeslein G, Morrison PJ, Chen Y, Ahsan H, Watson P, Lynch HT, de la Chapelle A, Fodde R, Pasche B: TGFBR I*6A may contribute to hereditary colorectal cancer. J Clin Oncol 2005, 23:3074-3078.

10. Skoglund J, Song B, Dalén J, Dedorson S, Edler D, Hjern F, Holm J, Lenander $\mathrm{C}$, Lindforss $U$, Lundqvist N, Olivecrona $\mathrm{H}$, Olsson L, Påhlman L, Rutegård J, Smedh K, Törnqvist A, Houlston RS, Lindblom A: Lack of an association between the TGFBRI*6A variant and colorectal cancer risk. Clin Cancer Res 2007, 13:3748-3752.

II. Kaklamani V, Baddi L, Rosman D, Liu J, Ellis N, Oddoux C, Ostrer H, Chen $Y$, Ahsan $H$, Offit $K$, Pasche $B$ : No major association between TGFBRI*6A and prostate cancer. BMC Genet 2004, 5:28.

12. You W, Liu Z, Zhao J, Zheng M, Zheng SY, Liu X, Zhang HT: No association between TGFBRI*6A and lung cancer. J Thorac Oncol 2007, 2:657-659.

13. Castillejo A, Rothman N, Murta-Nascimento C, Malats N, GarcíaClosas M, Gómez-Martínez A, Lloreta J, Tardón A, Serra C, GarcíaClosas R, Chanock S, Silverman DT, Dosemeci M, Kogevinas M, Carrato A, Soto JL, Real FX: TGFB I and TGFBRI polymorphic variants in relationship to bladder cancer risk and prognosis. Int | Cancer 2009, | 24:608-6|3.

14. Valle L, Serena-Acedo T, Liyanarachchi S, Hampel H, Comeras I, Li Z, Zeng Q, Zhang HT, Pennison MJ, Sadim M, Pasche B, Tanner SM, de la Chapelle A: Germline allele-specific expression of TGFBR I confers an increased risk of colorectal cancer. Science 2008, 32I:136I-1365.

15. Buhard O, Cattaneo F, Wong YF, Yim SF, Friedman E, Flejou JF, Duval A, Hamelin R: Multipopulation analysis of polymorphisms in five mononucleotide repeats used to determine the microsatellite instability status of human tumors. J Clin Oncol 2006, 24:24I-25I.

16. Zhang HT, Zhao J, Zheng SY, Chen XF: Is TGFBR I*6A really associated with increased risk of cancer? J Clin Oncol 2005, 23:7743-7744.

17. Castillejo A, Gomez A, Garrigos N, Guaraz P, Garcia M, Sanchez T, Barbera VM, Guillen C, Carrato A, Soto JL: TGFBRI is a target gene for frameshift mutations in colorectal tumors with microsatellite instability. Proceedings of the Clinical and Translational Oncology: 10-12 May 2007; Las Palmas, Islas Canarias. Spain 2007, 9(I):22-23.

\section{Pre-publication history}

The pre-publication history for this paper can be accessed here:

http://www.biomedcentral.com/1471-2407/9/193/pre pub 